\title{
Depistage Des Lesions Du Col Uterin Au Chu Tokoin De Lome : Resultats D’une Serie De 512 Cas.
}

\author{
Tchin Darre \\ Laboratoire d'Anatomie et Cytologie Pathologiques, CHU Tokoin, Lomé \\ Abdoul-Samadou Aboubakari \\ Services de Gynécologie, CHU Tokoin, et Kara \\ Yaovi Edem James \\ Laboratoire d'Anatomie et Organogenèse, CHU Tokoin, Lomé \\ Koué Folligan \\ Laboratoire d'Histologie-Embryologie, CHU Tokoin, Lomé
}

Bingo N'bortche

Services de Gynécologie, CHU Tokoin, et Kara

Koffi Amegbor

Laboratoire d'Anatomie et Cytologie Pathologiques, CHU Tokoin, Lomé

Koffi Akpadza

Services de Gynécologie, CHU Tokoin, et Kara

Gado Napo-Koura

Laboratoire d'Anatomie et Cytologie Pathologiques, CHU Tokoin, Lomé

doi: 10.19044/esj.2016.v12n12p129 URL:http://dx.doi.org/10.19044/esj.2016.v12n12p129

Abstract

Purpose: Cervical cancer remains a real public health problem in Togo. This study was undertaken to sensitize women and to determine the prevalence of precancerous and canrous lesions of the cervix in the Togolese woman.

Material and methods: This is a retrospective descriptive study of the results of a consecutive series of Pap smears performed in the Laboratory of Pathological Anatomy and Cytology CHU Tokoin Lome 2011-2013.

Results: Epidemiological, 512 FCV were interpretable. The average age of women was 37.6 years, extreme 19 and 87 years. By occupation, we noted a predominance of unemployed women (351cas, 68,6\%), followed by women traders (103 cases, $20,1 \%$ ) . The distribution by marital status was possible to observe a predominance of married women (234 cases, 45, $7 \%$ ). In terms of reproductive history, we found a mean gravidity and parity respectively 3,5 and

We noted 22 women living with HIV, 77.2\% were infected with HIV1. 
Cytopathological, 512 smears selected, we observed 44 abnormal smears (8,6\%). It was indeterminate lesions ASCUS / ASCUS (8 cases), endocervical hyperplasia (3 cases), low-grade lesions (17 cases), high-grade lesions (10 cases) and invasive carcinoma (6 cases). Conclusion: The detection rate of cervical cancer is low among the Togolese woman. The health professionals should take the habit of asking the FCV any woman having a consultation.

Keywords: Screening, Pap Smears, cervical cancer, HPV, Togo

\section{Résumé}

Objectif : Le cancer du col demeure un réel problème de santé publique au Togo. Cette étude a été entreprise pour sensibiliser les femmes et déterminer la prévalence des lésions précancéreuses et cancéreuses du col utérin chez la femme Togolaise.

Matériel et méthodes: Il s'est agit d'une étude rétrospective descriptive et transversale portant sur les résultats d'une série consécutive de FCV réalisés dans le Laboratoire d'Anatomie et de cytologie Pathologiques du CHU Tokoin de Lomé de 2011 à 2013. Les paramètres sociodémographiques, les antécédents médicaux, et les résultats du dépistage ont été étudiés.

Résultats : Au plan épidémiologique, 512 FCV étaient interprétables. L’âge moyen des femmes était de 37,6ans, les extrêmes 19 et 87 ans. Selon la profession, nous avons noté une prédominance des femmes sans emploi (351cas, 68,6\%), suivi des femmes commerçantes (103 cas, 20,1\%). La répartition selon le statut matrimonial avait permis d'observer une prédominance des femmes mariées (234 cas, 45,7\%). Au niveau des antécédents reproductifs, nous avons révélé une gestité et une parité moyenne respectivement de 3,5 et 2. Nous avons noté 22 femmes séropositives, dont 77,2\% étaient porteuses du VIH 1.

Au plan cytopathologique, sur les 512 frottis retenus, nous avons observé 44 frottis anormaux (8,6\%). Il s’agissait de lésions indéterminées ASCUS/ASCUS (8 cas), d’hyperplasie endocervicale (3 cas), des lésions de bas grade (17 cas), des lésions de haut grade (10 cas) et du carcinome invasif (6 cas).

Conclusion : Le taux de dépistage de cancer du col est faible chez la femme Togolaise. Les professionnels de la santé devraient prendre l'habitude de demander le FCV à toute femme se présentant à une consultation.

Mots clés : Dépistage, frottis cervico-vaginal, cancer du col, HPV, Togo 


\section{Introduction}

Le cancer du col demeure un problème de santé publique dans les pays en développement en général et au Togo en particulier. Il occupe le deuxième rang des cancers génitaux de la femme au plan mondial chez qui il représente 12\% des tumeurs malignes (OMS, 2007). En 2000, on comptait environ 470000 nouveaux cas, dont près de $80 \%$ dans les pays en développement (CURADO, 2007). Le principal facteur étiologique incriminé est le Papilloma Virus Humain (HPV) (HESSEL, 2009). Le HPV est à l'origine des lésions dystrophiques qui aboutissement à long terme (10-15 ans), tous au moins pour des lésions sévères, au cancer invasif du col (HESSEL, 2009). L’importance de cette infection est parfois aggravée par l'état immunitaire de l'hôte (MOODLEY, 2006). Dans les pays développés, on note une régression de la mortalité liée au cancer du col, qui se justifie par la mise en place du dépistage systématique et des traitements des lésions précancéreuses (BALAHA, 2011).

A ce jour, il n'existe pas de programme national de dépistage organisé du cancer du col utérin au Togo. Cette étude a été entreprise pour d'une part sensibiliser des femmes sur le dépistage du cancer du col utérin et d'autre part déterminer la prévalence des lésions précancéreuses et cancéreuses du col utérin chez la femme Togolaise.

\section{Patientes, Matériel et méthodes}

Il s'est agit d'une étude rétrospective descriptive et transversale portant sur une période de 3ans dans l'unique service d'Anatomie et Cytologie Pathologiques du CHU Sylvanus Olympio de Lomé. Ces cas ont été colligés à partir des données des registres dudit laboratoire. Ces registres comportaient le prescripteur du FCV, l’âge, les renseignements cliniques, le résultat cytopathologique. La classification utilisée dans ce travail est le système de Bethesda et le logiciel Epi-info a servit au traitement statistique des données. Les paramètres sociodémographiques, les antécédents médicaux, et les résultats du dépistage ont été étudiés.

\section{Résultats}

\section{Données épidémiologiques}

Nous avons retenu durant les 3 ans, 512 FCV interprétables. L’âge moyen des femmes était de 37,6ans, les extrêmes 19 et 87 ans. La figure 1 illustre la répartition des patientes selon l’âge montrant une prédominance dans cas dans la tranche d'âge de 31-40 avec 22\% des cas. Selon la profession, nous avons noté 351 femmes sans emploi (68,6\%), 103 femmes commerçantes $(20,1 \%)$ et 58 femmes salariées $(11,3 \%)$. La répartition selon le statut matrimonial avait permis d'observer une prédominance des femmes mariées (234 cas, 45,7\%) suivie des femmes veuves (182 cas, 35,5\%). 
Au niveau des antécédents reproductifs, nous avons révélé une gestité et une parité moyenne respectivement de 3,5 et 2. L'ensemble de ces données est résumé dans le tableau I.

Le profil professionnel des prescripteurs était dominé par les gynécologues (305 cas, 59,6\%) représenté par le tableau II.

\section{Données sur le VIH}

Les femmes qui avaient accepté le dépistage du VIH étaient au nombre de 176. Sur les 176 femmes dépistées au VIH, 22 femmes étaient séropositives. Presque que la totalité des femmes était porteur du VIH 1 soit $77,2 \%$, avec une connaissance de leur séropositivité d'au moins 1an dans 45,5\%. La majorité des femmes étaient sous traitement antirétroviral et la durée de ce traitement de moins d'1 an dans la plupart des cas. Le tableau III résume les informations sur le VIH.

\section{Données cytopathologiques}

Sur les 512 frottis retenus, nous avons observé 44 frottis anormaux (8,6\%). Il s’agissait de lésions indéterminées ASCUS/ASCUS (8 cas), d’hyperplasie endocervicale (3 cas), des lésions de bas grade (17 cas), des lésions de haut grade (10 cas) et du carcinome invasif (6 cas), comme le montre le tableau IV. Les lésions de bas grade étaient essentiellement des condylomes (16 cas). Les patientes étaient en moyenne dans la quarantaine pour des lésions dysplasiques et de dix années en moyenne plus âgées pour les cas de carcinomes invasifs. Le tableau $\mathrm{V}$ montre la répartition des cas de FCV pathologiques en fonction de l’âge moyenne et de la parité moyenne.

\section{Discussion}

\section{Discussion des données épidémiologiques}

Notre étude, sur les frottis de dépistage du cancer du col utérin a rassemblé 512 femmes dont 223 soit 43,6\% avaient entre 30 et 50 ans. Cette tranche d’âge représente la population cible dans le dépistage du cancer du col à cause de la forte prévalence des lésions cancéreuses chez ces femmes (HESSEL, 2009). Néanmoins, des cas ont été observés au delà de 60 ans, faisant préconiser la poursuite de la réalisation des FCV même après 50ans (HESSEL, 2009).

Nous constatons par conséquent que les prescripteurs de ces frottis qui étaient constitués de gynécologues-obstétriciens et des autres médecins étaient suffisamment avertis. Ces derniers, en effet, ne disposent pas de fiches incluant les données sur l'identité complète des patientes, ainsi que sur les antécédents gynécologiques, pour la prescription des frottis. En outre, les femmes ne bénéficiant pas souvent d'un nouvel interrogatoire au moment du prélèvement, n’ont pu donner d'informations sur la date des premières 
règles, l’âge au premier rapport sexuel, le nombre de partenaires sexuels, ainsi que leur statut matrimonial, avec comme conséquence la réduction de la taille de notre échantillon. Ces informations qui constituent des facteurs de risque importants de cancers du col, nous auraient permis d'avoir un meilleur aperçu sur les facteurs de risque dans notre pays.

L’âge moyen des femmes de notre série (37,6 ans), était comparable à celui rapporté par Claeys dans sa série (35,8 ans), mais plus bas que celui de la série Camerounaise (45,3ans) (CLAEYS, 2003 ; MBOUDOUD, 2008). Nous avons observé des cas avant 20 ans, d’où la nécessité d'initier le dépistage du cancer du col très précocement ; généralement après le début de l'activité sexuelle. Les données concernant la parité ne différaient pas de cellules des autres études confirmant sa place dans les facteurs de risques des cancers du col (MOODLEY, 2006; TEBEU, 2005). Le faible taux de nullipare ne devrait nullement nous faire songer à une exclusion de ces dernières du dépistage du cancer du col, puisque que des cas de lésions dysplasiques et un carcinome invasif ont été observés.

\section{Discussion des données sur le VIH}

Plusieurs études ont montré que si le risque de lésions précancéreuses aussi bien de bas que de haut grade était plus important chez les femmes VIH+, il n'en est pas de même des lésions cancéreuses (MOODLEY, 2006 ; CUBI, 2000). Dans notre série, la plupart des femmes étaient sous traitement Anti RetroViral (ARV). Ceci est encourageant car selon Robinson, le traitement ARV aurait un effet bénéfique sur l'évolution des lésions cervicales chez les femmes séropositives (ROBINSON, 2002 ; OUATARA, 2008). Il est recommandé que les femmes séropositives au VIH bénéficient de deux tests de dépistage du cancer du col utérin la première année, puis d'une surveillance annuelle.

\section{Discussion des données cytopathologiques}

Cette étude montre un pourcentage non négligeable $(8,6 \%)$ de patientes présentant sur leur frottis des anomalies; notre fréquence est légèrement supérieure de celle rapporté par Bennis au Maroc qui était de 6\% des cas (BENNIS, 2007).

Les lésions intra-épithéliales (CIN) de bas grade à savoir le condylome et les SILI constituaient la principale anomalie cytologique retrouvée soit 38,7\% des cas. Ce taux est inférieur à celui des études de Claeys, soit 78,4\%, mais comparable au taux rapporté par Mboudoud qui était de 35,4\% (CLAEYS, 2003 ; MBOUDOUD, 2008).

L’âge moyen (35,7 ans) des femmes porteuses de ces lésions était légèrement inférieur de celui rapporté au Cameroun qui était de 39 ans (MBOUDOUD, 2008). Les condylomes représentaient ces lésions de bas 
grade, Or ces derniers constituent une manifestation du HPV qui est retrouvée dans environ 94\% des dysplasies cervicales (RANABHAT, 2011). L’âge moyen de survenue de ces lésions concordait avec les données de la littérature (RANABHAT, 2011 ; SOLOMON, 2001).

Les lésions de haut grade venaient en seconde position soit 22,7\% des cas. Elles ont été rares chez les femmes jeunes, néanmoins un cas a été observé chez une patiente de 19 ans; nous faisant songer à une influence de certains facteurs tels que les infections à VIH et HPV (HESSEL, 2009). Le premier diminue l'âge de survenue des lésions dystrophiques et du cancer du col, surtout en cas d'association d'un HPV à haut risque, favorisant leur survenue précoce chez les femmes atteintes (CUBI, 2000).

Six cas de cancers invasifs, soit $13,6 \%$ des cas ont été observés. Ce taux était largement au dessus des 0,2\% rapporté dans l'étude de Claeys (CLAEYS, 2003). Ceci peut s'expliquer par l'âge relativement avancé de nos patientes allant jusqu'à 87 ans. Leur âge moyen était de 44,9ans, correspondant à l'âge moyen de survenue de cancer du col au Togo, soit 47 ans (DARRE, 2016). Par ailleurs, on notait un écart d'une dizaine d'année par rapport à l’âge moyen des lésions dystrophiques, ce qui correspond au délai d'évolution des lésions dystrophiques vers un cancer (CURADO, 2007 ; SOLOMON, 2001). La multiparité restait un facteur de risque considérable ; dans ce groupe la parité moyenne était de 6, supérieure aux parités moyennes des 2 précédents groupes. En outre, l’âge moyen au premier accouchement était relativement plus bas chez les patientes avec carcinome invasif comparés aux autres groupes. Ceci traduit une précocité des rapports sexuels chez ces patientes, facteur de risque connu dans la survenue du cancer du col (OMS, 2007 ; RANABHAT, 2011).

Les autres lésions sont de type ACUS/AGCUS et les hyperplasies endocervicales. Pour ces dernières, l’âge moyen de survenue était de 43,4 ans. Cette lésion, bien que son type n’ait pas été précisé dans notre cas, est souvent précurseur du cancer de l'endomètre dont l'âge moyen de survenue se situe entre 50 et 60ans (BEBY-DEFAUX, 2004).

\section{Conclusion}

Le cancer du col utérin reste le deuxième cancer de la femme au Togo, tant du point de vue de la morbidité que sur le plan de la mortalité. Ce qui interpelle la nécessité d'une politique de surveillance systématique des affections génitales instituant le dépistage à son stade initial à l'aide de l'examen par FCV. Le taux de dépistage de cancer du col utérin est faible chez les femmes togolaises, d’où l'impérieuse nécessité d'un programme national de dépistage organisé du cancer du col utérin dans notre pays. Les patientes de plus en plus jeunes étant atteintes de lésions cervicales sévères, il serait intéressant d'analyser la place du HPV et du VIH chez ces 
dernières ; Ces virus étant fortement en cause dans la survenue du cancer chez les patientes jeunes.

\section{Références:}

BALAHA MH, AL MOGHANNUM MS, AL GHOWINEM N, AL OMRAN S. Cytological pattern of cervical papanicolaou smear in eastern region of Saudi Arabia. J Cytol 2011; 28:173-7.

BEBY-DEFAUX A, BOURGOIN A, RAGOT S, BATTANDIER D, LEMASSON JM, RENAUD O, et al. Human papillomavirus infection of the cervix uteri in women attending a Health Examination Center of the French social security. J. Med Virol 2004; 73(2):262-8.

BENNIS S, MENIAR S, AMARTI A, BIGOU A. La place du frottis cervico-vaginal dans le diagnostic du cancer du col utérin au Maroc, region de Fès-Boulemane. J. Easter Mediterranean Health 2007; 3(5): 1153-9.

CLAEYS P, DE VUYST H, MZENGE G, SANDE J, DHONDT V, TEMMMERMAN M. Interrogation of the cervical screening in family planning clinics. Int J Gyn Obstet 2003; 81(1):103-8.

CUBI HA, SEAGAR AL, BEATTIE GJ, MONAGHAN S, WILLIAMS AR. A longitudinal study of HPV detection and cervical pathology in VIH infected women. Sex. Transm. Inf. 2000; 76:257-61.

CURADO MP, EDWARDS B, SHIN HR, STORM H, FERLAY J. Cancer Incidence in Five Continents. Vol IX. IARC Scientific Publications 2007, No. 160, Lyon, IARC Press.

DARRE T, WALLA A, KPATCHA TM, ABOUBAKARI AS, MANEH N, KOULINGA $M$, et al. Cancers in the Elderly seen in Anatomical Pathology Laboratory in Lome, Togo.

Open Journal of Pathology 2016; 6: 26-31.

HESSEL L. Introduction de la vaccination contre les papillomavirus humains dans les pays en développement : bilan et perspectives. Med Trop 2009; 69: 323-6.

MBOUDOUD ET, KKEGOUM B, NDOM P, ZAMBO H, ENOW O, DOH AS. Les frottis cervico-vaginaux à l'hôpital général de Yaoundé: Résultats d'une série de 1459 cas. Oncol Clin en Afr 2008; 4(1):11-5.

MOODLEY JR, HOFFMAN M, CARRARA H, ALLAN BR, COOPER DD, ROSENBERG L, et al. HIV and pre-neoplastic lesions of the cervix in the South Africa: a case-control study. BMC Cancer 2006; 6:135.

ORGANISATION MONDIALE DE LA SANTE. Lutte contre le cancer du col de l'utérus. Guide des pratiques essentielles. OMS Genève 2007.

RANABHAT SK, SHRESTHA R, TIWARI M. Analysis of abnormal epithelial lesions in cervical Pap smears in Mid-Western Nepal. J. Pathol Nepal 2011; 1:30-3. 
ROBINSON WR, FREEMAN D. Improved outcome of cervical neoplasia in VIH infected women in the era of highly active antiretroviral therapy. AIDS Patient Care 2002; 16: 61-5.

OUATTARA S, DAO B, BAMBARA M, SOME DA, DEMBELE A, SIOHO N. Dépistage du cancer du col utérin chez les femmes séropositives au VIH. Oncol Clin en Afr 2008 ; 4(1): 7-10.

SOLOMON D, DAVEY D, KURMAN R, MORIARTY A, O'CONNOR D, PREY M, et al. The 2001 Bethesda System: terminology for reporting results of cervical cytology. JAMA 2002; 24; 287(16):2114-9.

TEBEU PM, SANDJONG I, NKELE N, FOKOUA S, ACHU P, KOUAM $\mathrm{L}$, et al. Lésions précancéreuses du col utérin en zone rurale: Etude transversal. Med Afr Noire 2005; 52(1): 27-31.

Tableau I: Caractéristiques sociodémographiques.

- Moyenne

Age (ans)

- Extrêmes

Profession

- Sans emploi

- Commerçante

- Salarié

\section{Statut matrimonial}

- Mariée

- Veuve

- Célibataire

- Divorcée

\section{Gestitité}

- Moyenne

- Maximum

\section{Parité}

- Moyenne

35,7

19-87

$351(68,6 \%)$

$103(20,1 \%)$

$58(11,3 \%)$

$234(45,7 \%)$

$182(35,5 \%)$

$73(14,3 \%)$

23 (4,5\%)

- Maximum 
Tableau II: Répartition des patientes en fonction des prescripteurs.

\begin{tabular}{lcc}
\hline Caractéristiques & $\begin{array}{c}\text { Effectif } \\
(\mathrm{N}=512)\end{array}$ & $\begin{array}{c}\text { Fréquence } \\
(\mathrm{P}=100 \%)\end{array}$ \\
\hline Gynécologues & 305 & 59,6 \\
Résidents de gynécologie & 88 & 17,2 \\
Médecins généralistes et & 70 & 13,7 \\
Autres spécialités & & \\
Infirmiers & 22 & 4,3 \\
Malade elle-même & 15 & 2,9 \\
Non précisé & 12 & 2,3 \\
\hline
\end{tabular}

Tableau III: Informations sur le VIH.

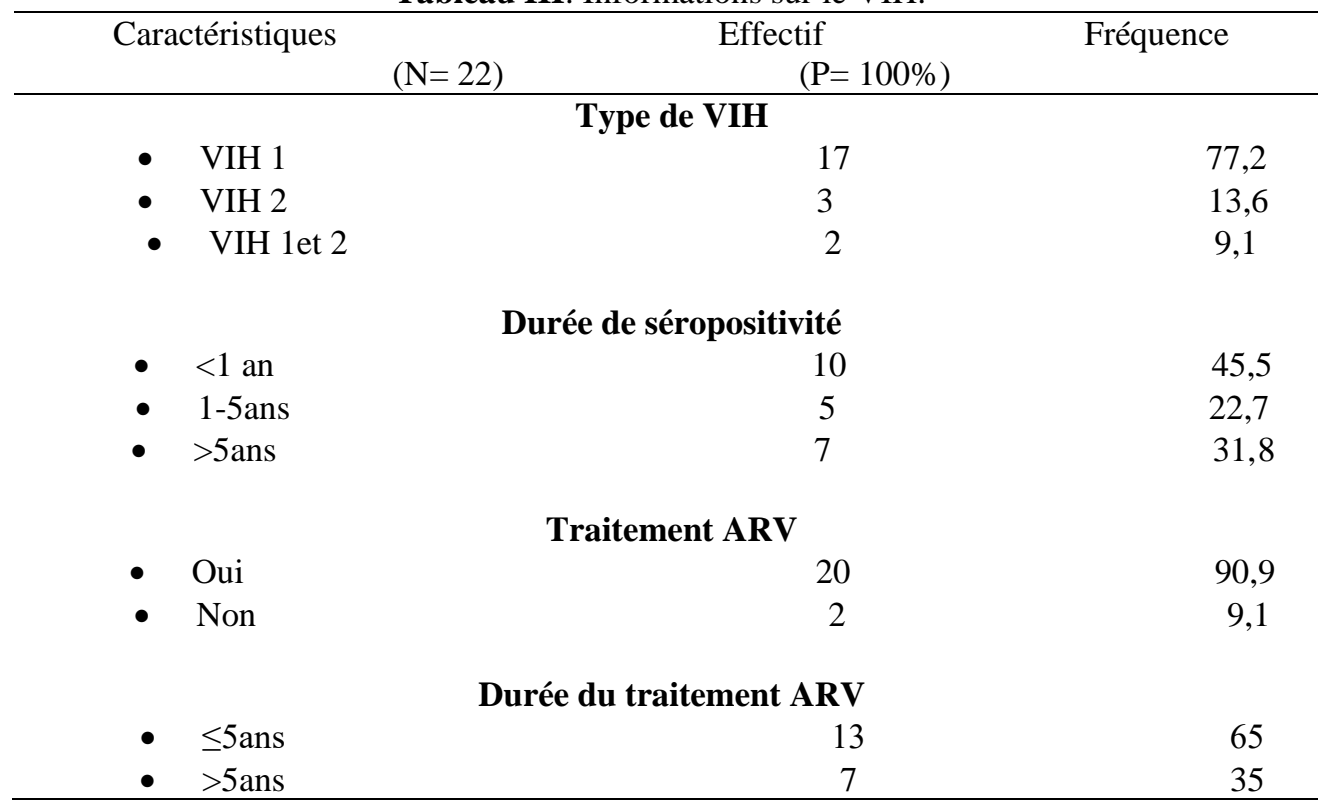

Tableau IV: Résultats cytopathologiques

\begin{tabular}{lcc}
\hline $\begin{array}{r}\text { Effectif } \\
(\mathrm{N}=44)\end{array}$ & $\begin{array}{c}\text { Fréquence } \\
(\mathrm{P}=100 \%)\end{array}$ & \\
\hline ASCUSS et AGCUSS & 8 & 18,2 \\
Hyperplasie de l'endocol & 3 & 6,8 \\
L-SIL (Condylome et CIN1) & 17 & 38,7 \\
H-SIL (CIN2 et CIN3) & 10 & 22,7 \\
Carcinome invasif & 6 & 13,6 \\
\hline
\end{tabular}


Tableau V: Moyenne d’âge, âge moyen au premier accouchement et parité moyenne selon le résultat cytopathologique

\begin{tabular}{lccc}
\hline \multicolumn{1}{c}{ Age moyen } & $\begin{array}{c}\text { Parité moyenne } \\
\text { accouchement }\end{array}$ & Age moyen au 1 & \\
\hline ASCUSS et AGCUSS & 29,2 & 3,2 & 19,7 \\
Hyperplasie de l'endocol & 43,4 & 5 & 19,1 \\
L-SIL (Condylome et CIN1) & 35,7 & 4,2 & 21,9 \\
H-SIL (CIN2 et CIN3) & 38,8 & 4,8 & 20,2 \\
Carcinome invasif & 44,9 & 6 & 18,7 \\
\hline
\end{tabular}

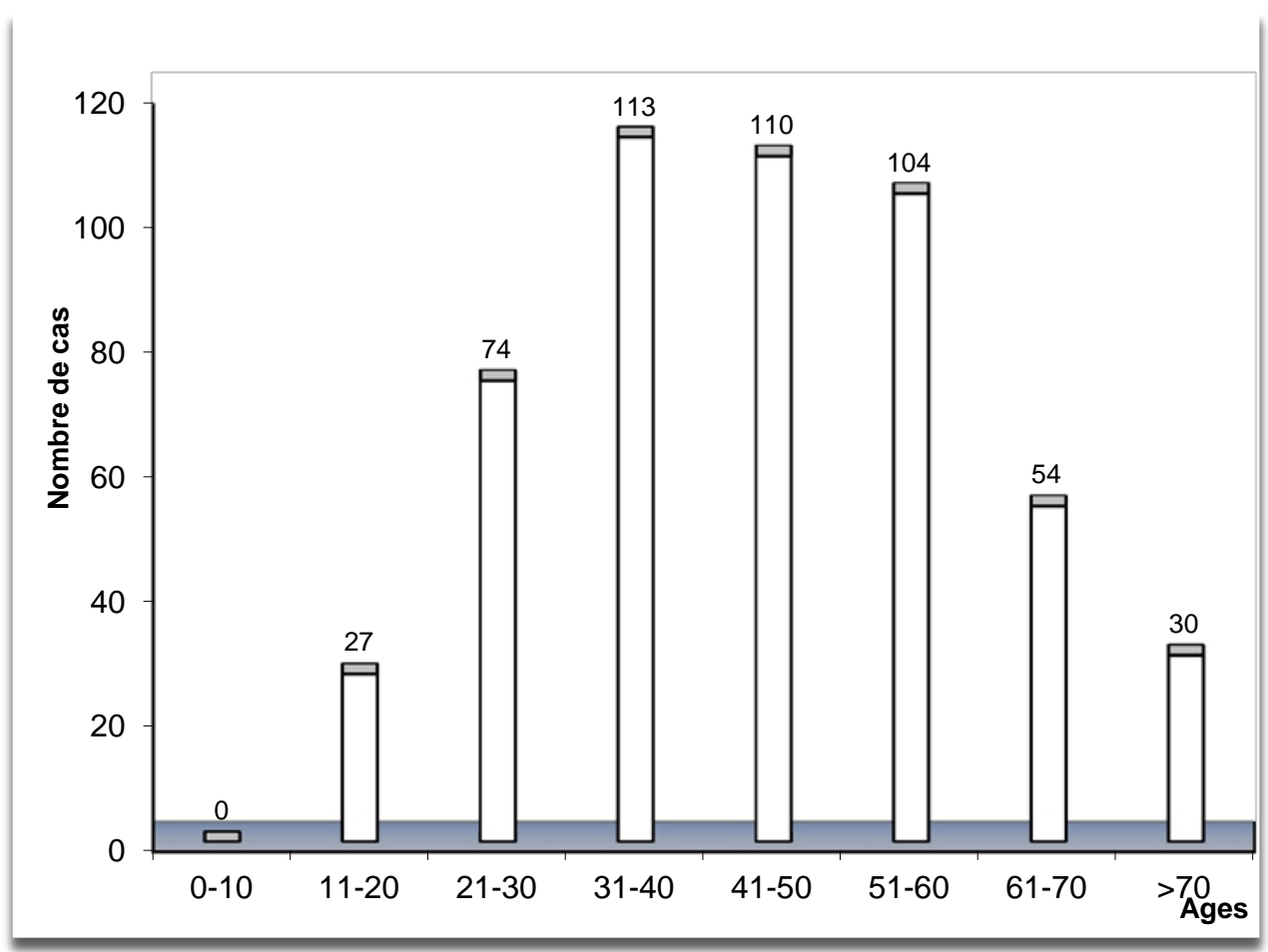

Figure 1 : Réparation des patientes en fonction de l'âge. 